\title{
Paralysis by Analysis: Choking, Clutching, and Reinvestment in Competitive Gameplay
}

\author{
Nicole Beres \\ Dept. of Computer Science \\ University of Saskatchewan \\ Saskatoon, SK, Canada \\ nicole.beres@usask.ca
}

\author{
Madison Klarkowski \\ Dept. of Computer Science \\ University of Saskatchewan \\ Saskatoon, SK, Canada \\ m.klarkowski@usask.ca
}

\author{
Regan Mandryk \\ Dept. of Computer Science \\ University of Saskatchewan \\ Saskatoon, SK, Canada \\ regan.mandryk@usask.ca
}

\begin{abstract}
Video games frequently invoke high-pressure circumstances in which player performance is crucial. These high-pressure circumstances are incubators for 'choking' and 'clutching'phenomena that broadly address critical failures and successes in performance, respectively. The eruption of esports into the mainstream has vitalized the need to understand performance in video games, and particularly in competitive games spaces. In this short workshop paper, we present a selection of findings and insights from a full paper (submitted for review) exploring potential mechanisms behind choking and clutching. We find that propensity to choke is positively predicted by trait reinvestmenta predisposition to 'focus inwards' in high pressure contexts, reverting to slower 'declarative' processing in lieu of more automated 'procedural' processing. We also find that propensity to clutch is positively predicted by player experience with competitive gaming. We propose that such findings can be utilized to scaffold and support performance in high-pressure gaming spaces, such as esports.
\end{abstract}

\section{CCS CONCEPTS}

- Human-centered computing Human computer interaction
(HCI); HCI design and evaluation methods; User studies

\section{KEYWORDS}

Esports, performance, video games, choke, clutch, competitive

\section{Introduction}

In competitive multiplayer video games, players are frequently under pressure. Whether that pressure manifests as a boss fight, a final dash to the finish line, or as the need for pixel-perfect shooting accuracy versus a skilled opponent, competitive games consistently demand that a player performs in high-pressure circumstances. While players often relish the experience of this challenge [13], little is known about the mechanisms that govern successful - or thwarted-performance in these contexts.

In esports, of particular relevance is the exploration of performance under pressure-an inescapable reality of competitive play. Colloquially understood in video game communities, and formalized in literature on physical sports
[1,18], are the concepts of 'choking' and 'clutching': respectively, failing and excelling in pressure conditions in which one's success is imperative. Although fundamental in competitive video game play, the phenomena of both choke and clutch have yet to be examined within the context of esports - a domain that consistently requires players to maintain optimal performance under high-pressure circumstances. We cannot assume that knowledge generated in the context of physical sports automatically transfers to the context of esports as they differ in critical ways (e.g., risk of injury, body contact), thus warranting specific investigation of these constructs in competitive multiplayer video games.

Despite a rise in esports, knowledge is still being formed about the experience of high-level play, and how success is achieved and maintained in these contexts. Without this knowledge, it is difficult for stakeholders and researchers to meaningfully support expertise development in esports contexts or guide best practice procedures. To address this, we turn to the application of selffocus models-i.e., models positing that choking is the result of attention focused inwards due to increases in anxiety [1] - that have been used in traditional sports to evaluate choke and clutch experiences. Comparative to traditional sports, performance in esports requires many of the same attentional demands, such as decision making, attention, memory, fine motor coordination, and game knowledge [21]. Applying self-focus models to the context of esports may provide insight into whether performance is influenced in the same ways as traditional sport.

Within this paper, we provide a selection of results and insights from a full paper (submitted for review) exploring the mechanisms that contribute to choke and clutch within competitive multiplayer video games. In particular, we report findings concerning tendency towards reinvestment, experience with competitive gaming, and choking and clutching prevalence.

\section{Background}

'Choking' is a term used to describe a scenario in which a player fails to maintain their performance in a high-pressure situation where it is expected or highly important [1]. In contrast, 'clutching' refers to a scenario wherein a player performs well under pressure in an unfavorable situation, leading to a desirable outcome [18]. While choking under pressure has been extensively studied in traditional sports, there is a comparative lack of 
research concerning clutch performances. However, within sports literature, attentional models of processing have received considerable support in conceptualizing the phenomenon of choke and clutch performance $[1,2,14]$. Performance in competitive multiplayer video games may also be affected by the same changes in attentional processes that lead to choke and clutch in traditional sport [21]. To this end, we explore the concept of 'reinvestment' and the related concepts of declarative and procedural processing - identified in the full paper as potentially contributive to choking and clutching in high-pressure contexts.

Declarative and Procedural Processes in Choking. The concepts of 'declarative' and 'procedural' processing describe psychological constructs that address task recall, knowledge, and automation. In cognitive psychology, declarative processes are described as slow and limited in nature, requiring attentional resources to consciously control the movement of a skill; conversely, procedural processes involved in performing a skill are automatic and do not demand attentional resources, thus enabling faster processing [22]. While developing a new skill (e.g., learning how to ride a bike), novices tend to utilize declarative processes to direct attention towards the systematic execution of a skill (e.g., pedalling, balancing, steering), which is honed into procedural execution with experience [7,22]. However, in high-pressure situations, performance of a well-learned skill may be harmed when declarative processes interrupt the procedural mechanisms by redirecting attention towards the execution of the skill [7].

Research suggests that these high-pressure situations are antecedents to the experience of choke, with choking occurring under circumstances that induce performance anxiety-such as goalkeeping against a penalty shoot-out $[1,16]$. This anxiety shifts attention away from the procedural execution of a task towards declarative processing of the task, hindering performance $[1,14,22]$. The translation of this to the context of video games is clear, wherein these high-pressure circumstances are facilitated as a matter of course; for example, in one-on-one combat against an opponent, in time pressure, or in boss fights. This is especially true for competitive esports contexts, wherein stakes are significantly increased and margin for error is significantly decreased - potentially rendering the majority of a match, or of a game, a high-pressure scenario.

Expertise and Performance. While declarative processes are involved in developing a new skill, they may have adverse effects on the performance of a well-learned skill. Drawing attention towards the explicit execution of a task was found to impair expert performance, but improve novice performance, in a batting task [7]; further, drawing attention away from the explicit execution impaired novice performance, as declarative processes are beneficial when learning a skill.

Ego investment also plays a role in expertise, with selfconsciousness borne from audience pressure impairing expert, but not novice, performance [10]. Players of varying levels of expertise may also experience differences in attentional focus within competitive gaming contexts.
Clutch Performance. While the phenomenon of choking has been explored, less is known about the psychological state athletes experience during a 'clutch' performance, particularly in the realm of competitive multiplayer video games. Interestingly, some research has reported that clutch states involve declarative processing - such as deliberate focus and conscious effort to raise performance-rather than being fully automatic [8,17]. While these findings appear to contradict conceptualizations of declarative processing in high pressure contexts, research suggests that expert performers are capable of strategically allocating conscious attention toward regulating their psychological state (e.g., increasing effort and concentration) while maintaining the procedural execution of motor skills [6,19]. In other words, skilled athletes focus declarative processes on controlling psychological states, rather than consciously attending to the execution of procedural motor skills.

\subsection{Reinvestment and Performance in Sports}

Although not yet explored in the context of competitive video gaming, the self-focus model of 'reinvestment' is a crucial concept in understanding performance under pressure in physical sports.

In line with self-focus models of attention, performance decrements in sport may be due to a predisposition to focus attention inwards through rehearsal of explicit rules (that is, declarative processing), disrupting the procedural processes of executing a skill $[14,15]$. Masters et al. propose a model for 'reinvestment' of controlled processing, which refers to a tendency to consciously control the step-by-step components of executing a skill through use of explicit rules [14]. The declarative use of these explicit rules demands attentional resources, which disrupts the procedural processes of a well-learned skill [15]. High 'reinvestors' have been found to be more likely to fail under pressure on a complex task than low reinvestors [14], and to use more explicit, task-relevant (e.g., declarative) knowledge than low reinvestors while performing - the allocation of conscious awareness towards the execution of a task.

The concept of reinvestment has traditionally been studied in sport, in which explicit rules govern the physical or motoric components of the activity; however, Masters et al. suggest that the exploration of reinvestment in "... skills such as chess, which involve no motoric component but provide a rich source of failure under pressure", presents a noteworthy avenue of investigation [14]. Despite this, no research to date has investigated reinvestment in non-physical sports. The self-focus model of reinvestment may be useful for understanding performance decrements in esports. For example, reinvesting players performing in first-person shooters may use explicit rules such as, "I must keep my crosshairs still and centered while I aim for the head of an opponent and left click the mouse to pull the trigger," which may elicit a disruption in their performance. We submit that the applicability of reinvestment to the esports domain represents a notable avenue for investigation, and may further 
Paralysis by Analysis: Choking, Clutching, and Reinvestment in Competitive Gameplay

strengthen understanding of human performance in digital contexts.

\section{Methods}

A nuanced understanding of human performance within multiplayer video games is critical for the academic scaffolding and support of esports. We propose that a vital component of this experience is that of performance under pressure. Alongside other concepts detailed in the full paper, we investigate the role of reinvestment in the propensity to choke and clutch in competitive multiplayer video games. To this end, the relevant research question is as follows:

RQ1: How does trait reinvestment predict propensity to choke and clutch?

To explore this, we conducted a quantitative analysis on data gathered in an online survey. Alongside other trait inventories (e.g., inventories used to assess traits or characteristics) reported in the full paper, we included an inventory assessing individual tendency towards reinvestment, an item exploring player experience level with competitive gaming, and a custom inventory exploring propensity to choke and clutch.

\subsection{Participants}

Participants were recruited from Amazon's Mechanical Turk (MTurk), which is an online marketplace that allows researchers to deploy studies through Human Intelligence Tasks (HIT) to diverse populations [3]. Participants were pre-screened on MTurk for those who identified as gamers, and reported experience playing competitive multiplayer video games. Ethics approval was obtained from the University of Saskatchewan, and participants were compensated \$6USD; the study took approximately 30 minutes to complete. Prior to reporting their experiences with choke and clutch, participants were provided the following definitions of to ensure comprehension of the terms:

Choke: “'Choking' is a term used to describe a scenario in which a player fails to maintain their performance in a situation where it is expected or highly important."

Clutch: "The term 'clutching' refers to a scenario wherein a player performs well under pressure in an unfavourable situation, leading to a desirable outcome."

The final sample consisted of data from 210 participants from Canada and the United States (men=141, women=68, nonbinary=1) aged 18 to $59(\mathrm{M}=33.9, \mathrm{Mdn}=32.5, \mathrm{SD}=8.3)$. In accordance with ethics approval requirements, data collected from this study cannot be publically shared.

\subsection{Measures}

3.2.1 Choking and Clutching Propensity. As there is no validated scale of the propensity to clutch or choke, we constructed a four-item 5-point Likert scale for each construct. Example items include, 'I often choke in competitive multiplayer games' and 'I fall flat in video game situations that demand I perform well' (for choke); and, 'I often clutch in competitive multiplayer games' and 'I thrive in video game situations that demand I perform well' (for clutch). Internal consistency of the items were considered good [5]: Choking: $\alpha=.80$; Clutching: $\alpha$ $=.80$.

3.2.2 Reinvestment Scale. The 20-item Reinvestment Scale [14] was designed to measure an individual's tendency to focus attention inwards. In line with previous studies [9,11,12], the original yes/no response format was replaced by a 5-point Likert type scale ranging from 1 (extremely uncharacteristic) to 5 (extremely characteristic). The use of this scale is reported to be a more reliable and valid measure [20]. In our sample, Cronbach's $\alpha=.87$, which is in line with previously reported values $(\alpha=.80$ : [14]).

\subsection{Data Analyses}

We conducted multiple regression analyses using reinvestment to predict the propensity to clutch or choke. Using hierarchical regressions, we controlled for age and self-rated experience (in the competitive video game they play most) by entering them in the first block and adding predictors of interest in the second block. We report standardized regression coefficients $(\beta)$ and p-values for individual predictors and $R^{2}$ values, $\mathrm{F}$, and $\mathrm{p}$-values for the regression models. SPSS 26 was used for all statistical tests on the quantitative data.

\section{Results}

As shown in Table 1, the model was significant for both choking and clutching. Experience negatively predicted choking, whereas it positively predicted clutching. Age did not significantly predict either choking or clutching. Trait reinvestment-i.e., a tendency to consciously control the step-by-step components of executing a skill through use of explicit rules - predicted propensity to choke, but not propensity to clutch.

\begin{tabular}{|c|c|c|c|c|c|c|c|c|c|}
\hline & \multicolumn{3}{|c|}{ Model } & \multicolumn{2}{|c|}{ Age } & \multicolumn{2}{|c|}{ Experience } & \multicolumn{2}{|c|}{$\underline{\text { Reinvestment }}$} \\
\hline & $R^{2}$ & $\mathrm{~F}$ & $p$ & $\beta$ & $p$ & $\beta$ & $p$ & $\beta$ & $p$ \\
\hline & 0.160 & 13.1 & $<.001$ & .086 & .188 & -.295 & $<.001$ & .283 & $<.001$ \\
\hline Clutching & .215 & 18.8 & $<.001$ & .065 & .306 & .465 & $<.001$ & -.067 & .286 \\
\hline
\end{tabular}

Table 1. Reinvestment: Multiple regression results with explained variance at the second level $\left(R^{2}\right)$, standardized regression coefficients $(\beta)$, and $p$ values for regressions predicting the propensity to choke and clutch, using age, experience, and trait reinvestment as predictors.

\section{Discussion}

To investigate the research question ("How does trait reinvestment predict propensity to choke and clutch?"), we explored predictive relationships between reinvestment and the propensity to choke and clutch. That reinvestment emerged as a positive predictor for choking, but not for clutching, confirms - in the context of video games - extant sports literature contending 
that 'high reinvestors' are more likely to underperform in highpressure scenarios [14]. As in physical sports, a predisposition in video games towards the re-adoption of declarative processing (disrupting faster, and more intuitive, procedural processes) may thwart performance in high-pressure events. Players predisposed to reinvestment may find themselves suddenly reliant on explicit rules in pressure scenarios; e.g., the earlier example of a reinvesting first-person shooter player who, in a critical moment, focuses on crosshair placement, knowledge about their weapon (e.g., spray patterns), and rehearses required inputs (formerly an automated task). As declarative processing requires additional attentional resources, switching to these explicit rules may slow, limit, and otherwise thwart performance. In a qualitative study by Fanfarelli [4], professional Overwatch athletes identified two overarching themes as important elements to professional play: game sense (e.g., survival, prediction, communication, and thoughtfulness); and mechanics (aim, ability usage, movement, positioning, and team-based synergies). As competitive multiplayer video games often require fast reaction and response time, and strategic decisiveness, any disruption to the execution of an in-game response may constitute a failure in performance.

Further, the identification of reinvestment as a predictor for choking in video games supports its presence in less motoric tasks. While previous literature has largely examined reinvestment in the context of physical activity (e.g., ball sports), there has been a dearth of literature exploring its application in predominantly mental or cognitively-driven activities. As such, this research supports that reinvestment is not solely an artefact of the physical domain, and that explicit mental rules may also be automatedand likewise susceptible to deautomization. Alternatively, it may be that the motoric requirements of video game play (e.g., reaction time vs. response time, wherein reaction time includes a motor component; or, fine motor skills associated with aim) are the components being automated and deautomized.

Experience. Consistent across all results, less experience was connected with a greater propensity to choke. Conversely, more experience was connected with a greater propensity to clutch. These relationships are intuitive: those with less experience may be more likely to make mistakes, and especially so under pressure, due to reduced familiarity with their preferred competitive video game (e.g., map layouts, tactics, combos, character skills, and so on). Furthermore, as novices tend to utilize slower declarative processes [7,22], choking may be more prevalent for less experienced players. In contrast, those with increased experience may be more equipped to succeed in pressure conditions due both increased game knowledge, and automated response through the procedural processing.

Age. Age did not play a role in choke and clutch, indicating that players' propensity to clutch and choke may be associated with trait susceptibilities, experience, and ability in the game, rather than by factors related to age (such as physical condition). As such, these findings do not suggest that performance in competitive multiplayer games is not related to age - but rather that propensity to choke (or performance under pressure) may not be.

\subsection{Implications}

Our work reveals implications for esports training and management. We suggest that collecting trait inventories from rostered players may allow an esports organization to determine, ahead of time, if a player may benefit from training, coaching, or familiarization with pressure scenarios. We suggest that training may be specialized to accommodate susceptibility to traits (e.g., susceptibility to reinvestment). For example, a player with susceptibility to reinvestment may benefit from a training regimen that encourages procedural processing in play. Further, organizational staff may implement general pressure training programs that familiarize players with performance in highpressure circumstances - thus equipping players with the ability to maintain, or excel, in contexts where their performance is critical.

\section{Conclusion}

In this paper, we investigated the role that reinvestment plays in both choke and clutch performance. Our work extends findings from traditional sports literature into the virtual realm of esports. We found that tendency towards reinvestment positively predicted propensity to choke. On the other hand, propensity to clutch was positively predicted by player experience with competitive gaming. Our results support that mechanics susceptible to deautomization in physical sports also translate to more cognitive domains such as online multiplayer video games. Research that continues to build upon player trait susceptibilities and concepts traditionally used in physical sports will equip researchers and esports performance personnel (e.g., coaches, managers, and players) with the tools required to address performance concerns. By unpacking the relationships between trait reinvestment and propensity to choke and clutch, our work takes a step toward helping esports enthusiasts - from amateur competitors through professional athletes - perform their best when under pressure.

\section{REFERENCES}

[1] Roy F. Baumeister. 1984. Choking under pressure: Self-consciousness and paradoxical effects of incentives on skillful performance. J. Pers. Soc. Psychol. 46, 3 (1984), 610-620. DOI:https://doi.org/10.1037/0022-3514.46.3.610

[2] Sian L. Beilock and Thomas H. Carr. 2001. On the fragility of skilled performance: What governs choking under pressure? J. Exp. Psychol. Gen. 130, 4 (2001), 701-725. DOI:https://doi.org/10.1037/0096-3445.130.4.701

[3] Michael Buhrmester, Tracy Kwang, and Samuel D. Gosling. 2011. Amazon's mechanical Turk: A new source of inexpensive, yet high-quality, data? $\begin{array}{llllll}\text { Perspect. } & \text { Psychol. Sci. 6, } & 1 & \text { (2011), } & 3-5\end{array}$ DOI:https://doi.org/10.1177/1745691610393980

[4] Joey R. Fanfarelli. 2018. Expertise in professional overwatch play. Int. J. Gaming Comput. Simulations $\quad 10, \quad 1 \quad$ (2018). DOI:https://doi.org/10.4018/IJGCMS.2018010101

[5] A. Field. 2013. Discovering Statistics using IBM SPSS Statistics. In Discovering Statistics using IBM SPSS Statistics. 297-321. DOI:https://doi.org/10.1016/B978-012691360-6/50012-4

[6] Andrew Geeves, Doris J.F. McIlwain, John Sutton, and Wayne Christensen. 2014. To Think or Not To Think: The apparent paradox of expert skill in music performance. Educ. Philos. Theory 46, 6 (2014), 674-691. DOI:https://doi.org/10.1080/00131857.2013.779214

[7] Rob Gray. 2004. Attending to the Execution of a Complex Sensorimotor Skill: Expertise Differences, Choking, and Slumps. J. Exp. Psychol. Appl. 10, 1 (2004), 42-54. DOI:https://doi.org/10.1037/1076-898X.10.1.42 
Paralysis by Analysis: Choking, Clutching, and Reinvestment in Competitive Gameplay

[8] Denise M. Hill, Sheldon Hanton, Nic Matthews, and Scott Fleming. 2010. A qualitative exploration of choking in elite golf. J. Clin. Sport Psychol. 4, 3 (2010), 221-240. DOI:https://doi.org/10.1123/jcsp.4.3.221

[9] Robin C. Jackson, Kelly J. Ashford, and Glen Norsworthy. 2006. Attentional focus, dispositional reinvestment, and skilled motor performance under pressure. J. Sport Exerc. Psychol. 28, 1 (2006), 49-68. DOI:https://doi.org/10.1123/jsep.28.1.49

[10] Charles E. Kimble and Jeffery S. Rezabek. 1992. Playing Games Before an Audience: Social Facilitation or Choking. Soc. Behav. Personal. an Int. J. 20, 2 (1992), 115-120. DOI:https://doi.org/10.2224/sbp.1992.20.2.115

[11] Noel P. Kinrade, Robin C. Jackson, and Kelly J. Ashford. 2010. Dispositional reinvestment and skill failure in cognitive and motor tasks. Psychol. Sport $\begin{array}{llll}\text { Exerc. } & 11, & 4 & (2010),\end{array}$ DOI:https://doi.org/10.1016/j.psychsport.2010.02.005

[12] Noel P. Kinrade, Robin C. Jackson, and Kelly J. Ashford. 2015. Reinvestment, task complexity and decision making under pressure inbasketball. Psychol. Sport Exerc. 20, March (2015), 11-19. DOI:https://doi.org/10.1016/j.psychsport.2015.03.007

[13] Derek Lomas, Kishan Patel, Jodi L. Forlizzi, and Kenneth R. Koedinger. 2013. Optimizing challenge in an educational game using large-scale design experiments. In Conference on Human Factors in Computing Systems Proceedings. DOI:https://doi.org/10.1145/2470654.2470668

[14] R. S.W. Masters, R. C.J. Polman, and N. V. Hammond. 1993. "Reinvestment": A dimension of personality implicated in skill breakdown under pressure. Pers. Individ. Dif. 14, 5 (1993), 655-666. DOI:https://doi.org/10.1016/01918869(93)90113-H

[15] J. P. Maxwell, R. S.W. Masters, and J. M. Poolton. 2006. Performance breakdown in sport: The roles of reinvestment and verbal knowledge. Res. Q. Exerc. Sport 77, 2 (2006), 271-276. DOI:https://doi.org/10.1080/02701367.2006.10599360

[16] Christopher Mesagno and Denise M. Hill. 2013. Definition of choking in sport: Re-conceptualization and debate. Int. J. Sport Psychol. 44, 4 (2013), 267-277. DOI:https://doi.org/10.7352/IJSP2013.44.267

[17] Christopher Mesagno and Daryl Marchant. 2013. Characteristics of Polar Opposites: An Exploratory Investigation of Choking-Resistant and ChokingSusceptible Athletes. J. Appl. Sport Psychol. 25, 1 (2013), 72-91. DOI:https://doi.org/10.1080/10413200.2012.664605

[18] Mark Otten. 2009. Choking vs. Clutch performance: A study of sport performance under pressure. J. Sport Exerc. Psychol. 31, 5 (2009), 583-601. DOI:https://doi.org/10.1123/jsep.31.5.583

[19] John Toner and Aidan Moran. 2014. In praise of conscious awareness: A new framework for the investigation of "continuous improvement" in expert $\begin{array}{lllll}\text { athletes. } & \text { Front. } & \text { Psychol. } & & \end{array}$ DOI:https://doi.org/10.3389/fpsyg.2014.00769

[20] Penny S Visser, Jon A Krosnick, and Paul J Lavrakas. 2000. Survey research. In Handbook of research methods in social and personality psychology. Cambridge University Press, New York, NY, US, 223-252.

[21] Michelle W. Voss, Arthur F. Kramer, Chandramallika Basak, Ruchika Shaurya Prakash, and Brent Roberts. 2010. Are expert athletes 'expert' in the cognitive laboratory? A meta-analytic review of cognition and sport expertise. Appl.

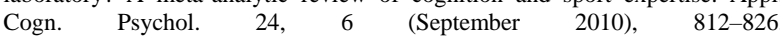
DOI:https://doi.org/10.1002/acp.1588

[22] A. Mark Williams, Keith Davids, and John Garrett Pascoe Williams. 2005 Visual Perception and Action in Sport. Routledge, New York, NY, USA. DOI:https://doi.org/10.4324/9780203979952 